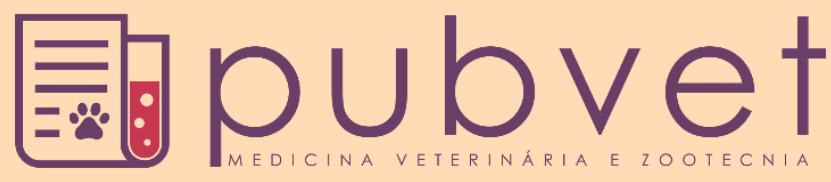

https://doi.org/10.31533/pubvet.v15n05a819.1-9

\title{
Cuidados paliativos em animais de companhia: Revisão
}

\author{
Natália Caroline Santos Almeida Magalhães ${ }^{1 * \bullet}$, Ana Luiza Dias Angelo ${ }^{2} \bullet$

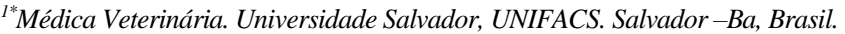 \\ ${ }^{2}$ Pós Doutora em Biotecnologia pela Universidade Federal da Bahia, UFBA. \\ *Autor para correspondência, E-mail: nataliacarolinemagalhaes@outlook.com
}

\begin{abstract}
Resumo. O tratamento paliativo é caracterizado por uma série de cuidados dedicados ao paciente sem chances de cura, como nos casos de diagnostico terminal ou doença crônica, amenizando as dores e os danos causados pela doença através de medidas terapêuticas que são selecionadas de acordo com a enfermidade em questão. Essa revisão de literatura tem como objetivo descrever quais os critérios para o tratamento paliativo em pequenos animais. Em situações em que os tutores desejam prolongar a vida do seu animal de estimação e, por isso, não consideram o uso da eutanásia como opção, os cuidados paliativos revelam sua efetividade ao promover o bem-estar e qualidade de vida ao paciente. A medicina paliativa é uma abordagem válida desde que a afecção não seja uma enfermidade de alto risco para saúde pública.
\end{abstract}

Palavras-chave: Bem-estar, dor, paliativo

\section{Palliative care in companion animals: Review}

\begin{abstract}
Palliative care is characterized by a series of patient care with no chance of cure, as in cases of terminal diagnosis or chronic disease, soothing the pain and damage caused by the disease through therapeutic measures that are selected according to the illness in question. This literature review aims to describe the criteria for palliative treatment in small animals. In situations where tutors want to prolong the life of their pet and therefore do not consider the use of euthanasia as an option, palliative care reveals its effectiveness in promoting well-being and quality of life for the patient. Palliative medicine is a valid approach as long as the condition is not a high-risk public health disease.
\end{abstract}

Keywords: Welfare, pain, palliative

\section{Introdução}

Mesmo sendo antiga, a relação ser humano-animal está crescendo cada vez mais ao longo dos últimos anos, progredindo não só em números, mas também no grau de afeto a estes. Segundo o Instituto Brasileiro de Geografia e Estatística (IBGE), em 2013, a quantidade de lares com cachorros foi maior do que com crianças (IBGE, 2013).

O termo "cuidados paliativos" é empregado para denominar a abordagem de uma equipe multiprofissional à pacientes fora das perspectivas terapêuticas de cura. A palavra "paliativa" é originada do latim palliun significando manto, proteção, isto é, proteger os doentes que já não mais integram a medicina curativa (Hermes \& Lamarca, 2013). Os cuidados paliativos específicos para animais ainda não são frequentes no Brasil; porém, são considerados uma tendência, observando a importância e o crescimento tanto no número de animais de estimação, quanto o avanço da própria Medicina Veterinária nacional (Lesnau \& Santos, 2013). Os cuidados paliativos destinam-se ao tratamento da dor, geriatria, amenizar os sinais de doenças crônicas que interferem na rotina do animal, casos de diagnóstico de doenças incuráveis e cuidados intensivos a longo prazo. Em países como os Estados Unidos, os tutores muitas vezes optam por essa abordagem por não considerarem a eutanásia como opção para seus 
animais, seja por questões culturais, religiosas, apego ou esperança de aumento da sobrevida do animal (Shearer, 2011b).

O objetivo desse trabalho é esclarecer sobre o tema e apontar os critérios para indicação do tratamento paliativo em pequenos animais.

\section{Histórico e definição}

O termo "cuidados paliativos" tornou-se mais conhecido na década de 60 , e consiste em cuidar dos pacientes com doenças crônicas, a fim de tratar a dor, diminuindo o sofrimento e maximizando as funções vitais. A prática de cuidar de peregrinos e viajantes nos abrigos ou hospedarias na antiguidade era caracterizado por "hospice" e confunde-se historicamente com cuidados paliativos, sendo o primeiro caracterizado por um movimento que engloba sugestões para potencializar o cuidado do paciente e tudo que o cerca, sobrepondo os cuidados paliativos (ANCP, 2012). O uso do termo "animal hospice", iniciou-se no final dos anos 80, quando os pioneiros Dr. Eric Clough e sua esposa Jane, que era enfermeira, em New Hampshire, o Dr. Guy Hancock, na Flórida, Dr. James Harris e Bonnie Mader, na Califórnia, começaram realizar cuidados paliativos independentemente em suas próprias práticas e, posteriormente, se uniram para apresentar dados sobre suas experiências em pequenos animais em várias conferências (Marocchino, 2011).

Nos Estados Unidos, médicos veterinários e profissionais de saúde mental reuniram-se para adaptar o tratamento paliativo a pacientes veterinários com base nos cuidados aplicados em seres humanos, tendo como diferencial a possibilidade de considerar a eutanásia como mais uma abordagem terapêutica (Shanan \& Balasubramanian, 2011).

\section{Normatização, princípios legais e circunstâncias éticas}

$\mathrm{Na}$ Medicina Veterinária não existem normas oficiais em relação aos cuidados paliativos, portanto, instituições e associações de classe se uniram para desenvolver suas próprias normativas e manuais com o propósito primordial de contribuir para o conhecimento, divulgação e aplicação dos tratamentos paliativos com o objetivo de melhorias na qualidade de vida destes animais e da relação humano-animal. São muitas instituições e associações que fazem parte desse movimento, a cada ano esse número aumenta, sendo o IAAHPC "International Association of Animal Hospice and Palliative Care" fundado em 2009, caracterizada como a associação responsável pela publicação das Diretrizes para Práticas Recomendadas em Animal Hospice e Cuidados Paliativos, publicado em 2014 e atualizado em 2017 (Cohen \& Regan, 2001).

Apesar do avanço e mudança na relação humano-animal, os animais ainda são considerados itens de propriedade pessoal (Shanan \& Balasubramanian, 2011). Só em 7 de agosto de 2019, senadores do Brasil, aprovaram em plenário, um projeto de lei que modificará a condição jurídica dos animais, de acordo com a proposta (PLC 27/2018), os mesmos começam a ter "natureza jurídica sui generis", ou seja, "sujeitos de direitos despersonificados". A ementa dispõe que os animais não sejam considerados coisas e se tornem respeitados como seres sencientes, o que denota que são compostos de natureza emocional e biológica e, desta forma, susceptíveis a dor e ao sofrimento (Cipriani, 2019). O tutor é legal e financeiramente responsável pelos cuidados do paciente, e o direito de tomar decisões relacionadas ao tratamento de um animal de companhia pertence exclusivamente ao seu tutor. Portanto, ao aceitar a intervenção terapêutica o responsável será submetido a uma entrevista de admissão hospitalar, e assinará um formulário de consentimento informado, afirmando estar ciente sobre todos os riscos e responsabilidades em relação aos cuidados paliativos (Shanan \& Balasubramanian, 2011).

A medicina paliativa para animais domésticos é considerada um tema pouco abordado dentro do curso de Medicina Veterinária no Brasil por se tratar de um assunto relativamente novo, porém existem disciplinas convencionais que tem a dupla função de habilitar o estudante para a compreensão sobre o tema (Lesnau \& Santos, 2013). São elas: anestesiologia, pois estuda o alívio da dor em intervenções cirúrgicas, exames diagnósticos ou doenças crônicas; terapia intensiva, já que aborda o cuidado necessário aos pacientes que necessitam de assistência e monitorização 24 horas; terapêutica, por ser a ciência responsável em selecionar a terapia mais adequada para o tratamento de cada enfermidade; e clínica cirúrgica, pela possibilidade de 
intervenção de forma instrumental ou manual no corpo do paciente com objetivo de tratar ou diagnosticar uma doença ou melhorar a função de órgãos (CFMV, 2010).

\section{Aplicabilidade dos cuidados paliativos}

As doenças que justificam o tratamento paliativo são: doenças oncológicas, insuficiência de sistemas e órgãos (rim, fígado, glândula endócrina e coração, por exemplo), osteoartrite e condições neurológicas progressivas. Para estes pacientes será elaborado um plano de tratamento, levando em consideração estágio da doença, grau de dor e sinais clínicos. Além destes, essa abordagem se estende também a animais geriatras, consistindo na instituição de medicamentos, dietas e ambientes adaptados, a fim de promover um final de vida com qualidade a esses animas (Shanan et al., 2013).

O objetivo dos cuidados paliativos é o tratamento da dor sem finalidade de cura e amenizar os sinais clínicos de doenças crônicas que interfiram na rotina do animal (Shearer, 2011a).

\section{Escala da qualidade de vida}

A escala de qualidade de vida foi elaborada com o intuito de auxiliar médico veterinário e tutor quanto a efetividade dos cuidados paliativos e quando pensar na eutanásia como alternativa. O objetivo principal da escala é a avaliação dos fatores básicos e necessários a saúde priorizando as cinco 5 liberdades que todo animal deve possuir caracterizando o bem-estar (livre de fome, sede e desnutrição; livre de doença, dor e ferimentos; livre de desconforto; liberdade para expressar o comportamento normal da espécie; livre de medo e angústia). Neste contexto, os parâmetros avaliados são: dor, fome, hidratação, higiene, felicidade, mobilidade e mais dias bons do que maus (Quadro 1). Cada critério avaliado pontua de zero a dez, sendo 10 classificação excelente e cinco aceitável. Somatório acima de 35 pontos indica que o animal está apto para integrar ou continuar com o tratamento paliativo (Villalobos, 2011).

Quadro 1. Escala da Qualidade de Vida. Adaptada de Villalobos (2011)

\begin{tabular}{|l|l|}
\hline Score & Critério avaliado \\
\hline $0-10$ & $\begin{array}{l}\text { DOR - O controle correto da dor e a capacidade do animal de respirar é a principal preocupação. A dor do animal } \\
\text { está devidamente controlada? O animal consegue respirar normalmente? O uso do oxigênio é necessário? }\end{array}$ \\
\hline $0-10$ & $\begin{array}{l}\text { FOME - O animal se alimenta suficientemente? É necessário o auxílio com a mão para o animal aceitar } \\
\text { melhor a comida? É indicado o uso de sonda? }\end{array}$ \\
\hline $0-10$ & $\begin{array}{l}\text { HIDRATAÇÃO - O animal está hidratado ou observa-se desidratação? Para pacientes que não bebem } \\
\text { água corretamente é necessário o uso de fluidoterapia subcutânea diariamente. }\end{array}$ \\
\hline $0-10$ & $\begin{array}{l}\text { HIGIENE - O animal é capaz de realizar suas necessidades fisiológicas distante de onde fica? Mantém o } \\
\text { asseio independente? A doença interfere na higiene do paciente? }\end{array}$ \\
\hline $0-10$ & $\begin{array}{l}\text { FELICIDADE - O animal apresenta ânimo e interesse? Está atento e se relaciona com a família, } \\
\text { brinquedos etc.? É um animal deprimido, solitário, ansioso, entediado ou medroso? Pode mover a cama } \\
\text { do animal para perto de atividades familiar? }\end{array}$ \\
\hline $0-10$ & $\begin{array}{l}\text { MOBILIDADE - O animal consegue se levantar sem auxílio? Necessita de alguma ajuda humana ou } \\
\text { mecânica? Tem vontade de passear? O paciente apresenta convulsões ou desiquilibra constantemente? }\end{array}$ \\
\hline $0-10$ & MAIS BONS DIAS QUE MAUS DIAS \\
\hline
\end{tabular}

É considerado mais bons dias que maus dias, quando o animal se distancia da família constantemente, significa que a qualidade de vida pode estar comprometida. Dias considerados ruins são aqueles em que o animal apresenta com frequência: náusea, êmese, apatia, convulsões e ataxia, ou quando a doença se agrava, como nos casos de caquexia em neoplasias, fraqueza decorrente da anemia profunda, desconforto causado pela compressão/ obstrução tumoral gradual. Esses sinais indicam que a relação humano-animal não está sendo mais viável, contudo, se o tutor não aceitar a eutanásia cabe ao médico veterinário respeitar a decisão do cliente (Villalobos, 2011).

\section{Ambiente e avaliação contínua do paciente}

Ao aderir ao tratamento paliativo o tutor e o médico veterinário devem entrar em um acordo para garantir que o paciente viva e seja cuidado da melhor forma possível e em um ambiente confortável. O profissional responsável pode sugerir que sejam adaptados ao lar equipamentos para facilitar a vida do pet, como pisos emborrachados a fim de evitar lesões, rampas, portões, cama confortável e adequada 
para a condição do paciente, adotar métodos para manter a higiene e limpeza em torno do animal. Monitoramento da temperatura ambiente, som, umidade e iluminação devem ser convenientes para potencializar o conforto do animal (Shanan et al., 2013). É indispensável gerenciar a condição clínica e as necessidades físicas do paciente (August, et al., 2017).

O tutor é instruído a reconhecer sinais clínicos em seu animal, como náuseas, desidratação, dor, dispneia, infecções, obstruções urinárias, constipação, melena e anemia, pois esses sinais interferem no bem-estar do paciente. É importante que o responsável comunique toda e qualquer mudança sobre o estado de saúde do paciente, para que o médico veterinário providencie os métodos necessários, para evitar que se prolongue o sofrimento do animal (Shearer, 2011a).

Se o estado do paciente for estável, essas avaliações devem ser feitas semanalmente por um técnico da equipe ou médico veterinário, recolhendo dados necessários e amostras para documentar e acrescentar modificações no tratamento paliativo daquele animal. Se a condição do paciente for instável significa que as variações dos sinais clínicos ocorrem constantemente, portanto, as avaliações devem ser feitas diariamente, em algumas situações mais de uma vez ao dia. Assim, o tutor deverá ser treinado a fazer esse procedimento de avaliação, criando um relatório para ser entregue ao médico veterinário responsável pelo caso, para mudanças no tratamento ou para a decisão da eutanásia (Shanan et al., 2013).

Neste contexto, o veterinário deve orientar o tutor sobre o quadro da enfermidade do paciente e sobre o processo de morte, compartilhando as etapas do processo da doença de forma objetiva e concreta. Essas discussões incluem esclarecimentos sobre a doença e sua trajetória; e sobre as terapias paliativas disponíveis para o caso, dessa forma, equipe médica e tutor podem chegar a um consenso sobre as medidas a serem adotadas em prol da melhoria da qualidade de vida e bem-estar do animal (Shearer, 2011b).

\section{Trajetória das doenças}

Foi elaborada na medicina humana com o objetivo de auxiliar na precisão do tratamento paliativo, acompanhar alterações do paciente e facilitar na escolha de medicamentos de acordo com o curso da doença. Há quatro diferentes trajetórias da medicina humana que são utilizadas na Medicina Veterinária. A trajetória das doenças é dividida em períodos, que mensurará o tempo que o animal doente alcançará a debilidade, o curso, que definirá como o animal viverá, a qualidade de vida e estrutura antes da morte (Cohen \& Regan, 2001; Shearer, 2011b).

As neoplasias são doenças classificadas de curto período de declínio, com curso de boa saúde até uma rápida e previsível debilidade. Doença renal crônica, insuficiência hepática e insuficiência cardíaca congestiva, são distúrbios de lento período de declínio, no curso dessas doenças ocorre o aumento e intensidade dos sinais clínicos, e o animal nunca recupera sua saúde. Degeneração mielopática e disfunções cognitivas são patologias de período prolongado, necessitando de cuidados progressivos durante o curso devido a complicações secundárias, como lesões por pressão, úlceras de decúbito e infecções do trato urinário. Por fim, as lesões circulatórias ou neurológicas graves e súbitas, traumas, hérnia de disco intervertebral e isquemia cerebral são enfermidades de período súbito, que no decorrer do curso o animal apresenta mobilidade gravemente comprometida ou incapacidade funcional (Cohen \& Regan, 2001; Shearer, 2011b).

Quadro 2. Trajetória das doenças

\begin{tabular}{|c|c|c|c|}
\hline & & Períodos & Cursos \\
\hline & & Declínio curto & Boa saúde até uma rápida debilidade \\
\hline $\begin{array}{l}\checkmark \\
\checkmark \\
\checkmark\end{array}$ & $\begin{array}{l}\text { Doença renal crônica; } \\
\text { Insuficiência hepática; } \\
\text { Insuficiência cardíaca congestiva }\end{array}$ & Declínio lento & $\begin{array}{c}\text { Aumento e intensidade dos sinais } \\
\text { clínicos (o animal nunca recupera sua } \\
\text { saúde) }\end{array}$ \\
\hline$\checkmark$ & $\begin{array}{l}\text { Degeneração mielopática; } \\
\text { Disfunções cognitivas }\end{array}$ & Declínio prolongado & $\begin{array}{l}\text { Cuidados progressivos durante o } \\
\text { curso, cercado de lesões secundárias }\end{array}$ \\
\hline $\begin{array}{l}\checkmark \\
\checkmark \\
\checkmark \\
\checkmark\end{array}$ & $\begin{array}{l}\text { Lesões circulatórias ou neurológicas graves ou súbitas; } \\
\text { Traumas; } \\
\text { Hernia de disco intervertebral; } \\
\text { Isquemia cerebral }\end{array}$ & Declínio súbito & $\begin{array}{c}\text { Mobilidade gravemente comprometida } \\
\text { ou incapacidade funcional }\end{array}$ \\
\hline
\end{tabular}




\section{Terapias de suporte}

\section{Medicações e métodos para amenizar a dor}

No tratamento paliativo o ponto principal é o controle da dor do paciente terminal, sendo necessário recorrer a terapias que auxiliarão nesse quesito. A identificação não só da dor como o grau apresentado é crucial. Dores leves, o animal pode não demonstrar sinais, nas dores moderadas a graves podem ser observados apatia, anorexia, taquicardia, taquipneia, entre outros (Garcia et al., 2016). As terapias são classificadas em medicamentosas, com uso de fármacos, métodos fitoterápicos, e não medicamentosa (Shanan et al., 2013).

Como métodos farmacológicos para atenuar dores leves a moderadas podem ser utilizados os opioides de baixa potência e os anti-inflamatórios não esteroides. Para o controle de dores moderadas a graves são utilizados os opioides potentes, como a morfina (Garcia et al., 2016), e o fentanil considerado alternativa excelente para analgesia, mais potente que a morfina, apresenta-se na forma adesiva, permite facilidade em administrar e manter, não invasivo, promovendo diminuição dos efeitos colaterais e baixo custo (Aleixo et al., 2017). Os fitoterápicos são medicamentos originados de plantas medicinais, que além de amenizar a dor, melhoram a função dos órgãos, a exemplo da silimarina (Silybum marianum) que funciona como protetor hepático, a meadowsweet (Filipendula ulmaria) que apresenta efeito instantâneo na dor e inflamação. Dentre as técnicas não medicamentosas que amenizam as dores podem ser citadas as modalidades fisioterapêuticas como a massoterapia, laser ou ultrassom terapêutico, e acupuntura que se combinadas com medicamentos proporcionam o uso de doses mais baixas, minimizando os efeitos colaterais (Shanan et al., 2013).

O processo para amenizar as dores severas deverá ser tratado somente por um médico veterinário. A maioria dos pacientes paliativos estão sobre cuidados domiciliares, portanto, é importante a adesão de um "kit para conforto de emergência" que é constituído por medicamentos selecionados de acordo com o diagnóstico e patologia do animal em questão, com intuito de controlar a crise de dor até que o paciente esteja sob os cuidados veterinários (August et al., 2017).

\section{Suporte nutricional}

Se o paciente, mesmo enfermo, apresenta bom apetite, uma dieta, formulada de acordo com a doença apresentada é indispensável, porém se o animal já não tem mais interesse na comida, a variação do cardápio com alimentos altamente palatáveis se faz necessária (Shearer, 2011b). Além de oferecer pratos saborosos e da preferência do paciente, é importante ser ofertados em ambiente calmo e confortável. O uso de drogas que aliviam as náuseas e consequentemente estimulam o apetite do animal pode ser indicado, como por exemplo a metoclopramida e ondansetrona (Garcia et al., 2016).

O uso de sondas enterais (nasogástrica, esofágica e gástrica) torna-se necessário nos casos de doenças em que o animal mesmo com fome não consegue ingerir a comida (Shearer, 2011a).

\section{Cirurgia paliativa}

Tem como objetivo o alívio da dor, contendo os sinais clínicos, revitalizando o animal e melhorando a qualidade de vida, porém a cirurgia só deve ser adotada na ausência de outro procedimento menos invasivo, e com a certeza de que o método cirúrgico irá amenizar os sinais clínicos. A intervenção pode ser eficaz nos casos de osteossarcoma apendicular ou tumores com capacidade de metástase elevado e tumores ulcerados, onde a cirurgia reduz a chance de infecção sistêmica ou local (Garcia et al., 2016).

\section{Quimioterapia paliativa}

É o uso de fármacos que provocam danos químicos a células em crescimento e segmentação, contudo, esses medicamentos quimioterápicos não são capazes de diferenciar as células malignas das benignas, tornando toda célula em divisão vulnerável a quimioterapia (Gustafson \& Page, 2013). O tratamento quimioterápico paliativo pode ser indicado em casos de tumores mamários, sarcomas de tecidos moles, neoplasias multicêntricas e tumores que a retirada cirúrgica não é possível (Garcia et al., 2016). 
A quimioterapia por ser um método citotóxico, é cercado de efeitos colaterais, como vômitos, náuseas, diarreias, imunossupressão, por conta da supressão da medula óssea, ocasionando diminuição na contagem de leucócitos; e perda de pelos, principalmente na região da face e da cauda, voltando a normalidade ao cessar o tratamento quimioterápico. A gravidade dos sinais podem ser leve, moderado ou grave, sendo a maioria dos casos leves. Nas infecções graves é necessário a hospitalização do paciente para tratamento intensivo com o auxílio de antibioticoterapia e fluido intravenoso (Polton, 2008).

\section{Radioterapia paliativa}

Consiste na utilização de radiação ionizante para o tratamento local de tumores sólidos, não tendo eficácia sistêmica, atingindo somente células que estão sob o campo de radiação, portanto, os efeitos colaterais são mínimos (Larue \& Gordon, 2013). O uso da radioterapia é eficiente nos casos em que a neoplasia provoca dor constante, tumores comprimindo estruturas vitais ou bloqueando as vias aéreas, tumores orais, sarcomas de tecidos moles e tumores da tireoide (Garcia et al., 2016).

\section{Eficácia dos cuidados paliativos}

O que confere o sucesso do tratamento paliativo não é a cura, já que se aplica a pacientes crônicos ou terminais, mas, oferecer os cuidados necessários no momento certo para obter bons resultados ao tratar as dores e outros sinais clínicos ocasionados pela doença. Dessa forma, o tratamento paliativo é considerado bem sucedido quando há habilidade nos serviços prestados pelo médico veterinário, satisfação do tutor, conforto e bem-estar do paciente (August et al., 2017).

A utilização do tratamento paliativo, envolve a educação e o preparo sobre o processo da morte. A partir de um diagnóstico terminal a estimativa do período de vida do animal durante o tratamento serve como uma direção, mas não é capaz de determinar com exatidão o tempo que o paciente terá de vida, portanto, é necessário a observação constante do mesmo (Shearer, 2011b).

É importante preparar o tutor e até mesmo os familiares envolvidos e alertá-los que a morte do animal está próxima, sendo ela de forma natural ou por intermédio da eutanásia (Lesnau \& Santos, 2013). Devese descrever o processo com base na trajetória da doença em que o paciente está envolvido, abordar que os sinais da enfermidade e o processo da morte variam de acordo com a doença e a condição metabólica do animal, porém, existem os denominados "sinais da morte", são eles: anorexia, sonolência, fraqueza extrema, extremidades frias, respiração superficial e irregular e aumento de ruído respiratório. O tutor deve estar ciente e entender que esses sinais indicam a proximidade do óbito do animal (Shearer, 2011b).

\section{Plano de avaliação emocional}

Para um tutor investir nos cuidados paliativos, significa que existe um sentimento muito forte por seu animal de estimação, pois, além do gasto financeiro, requer o desgaste emocional ao presenciar todos os dias o sofrimento deste. O médico veterinário responsável pelo caso deve orientar o tutor a procurar auxílio de um psicólogo para conseguir lidar com as questões psicoemocionais, no entanto, o plano de avaliação emocional é mais uma ferramenta disponível no tratamento paliativo, composto por um conjunto de perguntas, que devem ser feitas de forma sutil e sincera pelo profissional veterinário dentro do consultório assim que o responsável recorrer aos cuidados paliativos. O questionário tem como objetivo avaliar o emocional do tutor em relação ao que ele pensa sobre o estado de saúde do seu animal, oferecer suporte afetivo, e ajudar a equipe veterinária a agir com cautela, respeitar e reconhecer o laço humano-animal (Cohen \& Regan, 2001).

O questionário ajuda o profissional a obter informações muitas vezes omitidas e facilita a relação entre o médico veterinário e o responsável. As perguntas subjetivas permitem que o profissional avalie quão envolvido sentimentalmente o tutor está no caso, além de serem avaliadas a aparência física do animal e a do tutor, a ligação que existe entre eles, e o quanto o processo da doença irá afetar a vida dos familiares do paciente, já as objetivas servem para avaliar como é a relação humano-animal, se existe outros animais de estimação na casa, se o paciente dorme na cama, se é como uma criança para o tutor, e qual é o grau de apego ao pet (Villalobos, 2011). Portanto, as perguntas subjetivas e objetivas têm o intuito de reunir dados sobre a vida pessoal e situação emocional do responsável (Cohen \& Regan, 2001). 
A avaliação é o instrumento que reúne as informações coletadas nas duas primeiras etapas do questionário (subjetivo e objetivo) e considera se há algum choque emocional na situação, se o tutor e familiares tem condições de entender e aceitar o processo de doença que o animal irá percorrer e o processo da morte. Finalmente, a etapa do planejamento, considerada a fase mais difícil, na qual o médico veterinário irá desenvolver um plano de apoio apropriado, encaminhando o tutor a uma ajuda psicológica (Villalobos, 2011).

\section{Eutanásia x ortotanásia}

Eutanásia, do grego "eu" - bom - e "thanatos" - morte - consiste no modo humanitário de tirar a vida do animal, sem dor e com mínimo estresse, no intuito de atenuar ou evitar o sofrimento. A prática fundamenta-se na intervenção humana com o objetivo de finalizar a vida do paciente (Felix et al., 2013; CONCEA, 2015). Já a ortotanásia não provoca; porém não evita, tampouco retarda o óbito. A técnica também promove uma morte sem sofrimento, mas isenta o profissional de utilizar recursos que prolonguem a vida, como a ventilação artificial, ressuscitação cardiopulmonar ou outros métodos invasivos (Cohen \& Regan, 2001; Felix et al., 2013).

\section{Cuidados paliativos e eutanásia: Promoção do bem estar}

Ao abordar sobre "bem-estar animal" o primeiro a se pensar são nos animais de abate, mas é uma pauta que se estende à todos os animais, incluindo os de companhia, portanto, os cuidados paliativos são promoção do bem estar, que garante o direito de vida com qualidade ao animal de estimação com diagnostico terminal ou crônico (August et al., 2017).

A eutanásia é também considerada uma promoção do bem-estar animal (CFMV, 2012), a técnica tem o objetivo de findar o sofrimento animal de forma irreversível. Como ingerência médica é considerada uma opção amplamente aceita na Medicina Veterinária, praticada em cuidados paliativos, mas não deve ser a primeira linha de tratamento da dor, tampouco substituir os cuidados paliativos, sendo obrigação do médico veterinário diante de um diagnóstico terminal, orientar o tutor sobre as opções de paliativos disponíveis para o caso (Shanan et al., 2013).

A opção de utilizar a eutanásia, ocorre somente quando se esgotam as alternativas de manutenção de qualidade de vida ou quando o tutor decidir não prosseguir com o tratamento paliativo. Quando a relação humano-animal não está sendo saudável, como nos casos em que os sinais da doença se tornam mais intensos, interferindo diretamente no bem-estar animal, a qualidade de vida se torna precária, os medicamentos não estão sendo eficazes, é possível pensar na hipótese da eutanásia, porém, alguns tutores possuem crenças e culturas as quais não aceitam a eutanásia como opção, portanto, cabe ao profissional respeitar a decisão do tutor e prosseguir com o tratamento paliativo (Villalobos, 2011).

Ao escolher a eutanásia, o preparo da técnica consiste em priorizar o conforto do animal, de preferência que o processo seja realizado no lar do paciente, eliminando o transtorno de mover um animal debilitado para o ambiente clínico. Quando a eutanásia é realizada no hospital ou clínica, é importante que o ambiente seja confortável, tranquilo, visando promover o bem-estar do animal, nos seus últimos momentos e priorizando a privacidade dos familiares que passarão por esse difícil acontecimento (Cooney et al., 2012).

\section{Fadiga por compaixão (Síndrome de Burnout)}

Profissionais que lidam com o sofrimento alheio constante, estão sujeitos a desenvolver um fenômeno que se caracteriza por um cansaço físico e emocional causado pela compaixão vivenciada com os pacientes que estão sofrendo física e mentalmente. Esses profissionais, mesmo que de forma involuntária transferem para si as dores, medos e sofrimento, resultando respostas somáticas e/ou defensivas em relação ao seu trabalho (Lago, 2008).

A definição desse transtorno se deu por médicos que trabalham com pacientes humanos, porém, os profissionais veterinários ao seguirem a área da medicina paliativa, estão expostos a fadiga por compaixão devido ao desejo natural humano em auxiliar o animal que padece, incluindo o fato de presenciar o sofrimento do tutor e família. Os sintomas apresentados na fadiga por compaixão podem ser depressão, estresse e trauma. Portanto, é importante que os profissionais que trabalham em cuidados 
paliativos mantenham consultas com psicólogos frequentemente, a fim de evitar e/ou amenizar os efeitos desse fenômeno (Shanan et al., 2013).

\section{Considerações finais}

A medicina paliativa envolve uma série de terapias selecionadas para o controle da dor e outros sintomas decorrentes de uma afecção crônica, com a finalidade de promoção do bem-estar animal. Aderir aos cuidados paliativos não significa abolir totalmente a eutanásia, apenas não é a única opção em casos de um diagnóstico terminal. O tema é extremamente relevante visto que os animais têm vivido mais e desenvolvido mais doenças de curso crônico, portanto, promover qualidade de vida a estes fiéis companheiros em seu curso final tem grande importância dentro de um seio familiar. Existem poucos trabalhos nacionais sobre esta temática, havendo assim carências de protocolos já estabelecidos e validados para realidade do mercado pet brasileiro.

\section{Referências}

Aleixo, G. A. S., Tudury, E. A., Coelho, M. C. O. C., Andrade, L. S. S., \& Bessa, A. L. N. G. (2017). Tratamento da dor em pequenos animais: classificação, indicações e vias de administração dos analgésicos (revisão de literatura: parte II). Medicina Veterinária, 11(1), 29-40. DOI: https://doi.org/10.26605/medvet-n1-1596

Academia Nacional de Cuidados Paliativos (ANCP). Manual de Cuidados Paliativos. ampl. E atual. Porto Alegre: Sulina, 2012.

August, K., Shanan, A., August, K., Cooney, K., Hendrix, L., Mader, B. \& Pierce, J. (2019). Animal Hospice and Palliative Care Guidelines. Published by the International Association of Animal Hospice and Palliative Care, mar. 2017. Disponível em: <www.iaahpc.org> Acesso em: 16 mar. 2019.

CFMV. Constituição (2012). Lei $n^{\circ}$ 1000, de 11 de maio de 2012.: Conselho Federal de Medicina Veterinária. Brasil. Disponível em: $<$ https://www.feis.unesp.br/Home/comissaodeeticaeusoanimal/resolucao-1000-11-05-2012-cfmv_-eutanasia.pdf > acesso em: 24 mar. de 2020.

CFMV. (2010). Cursos de Graduação de Medicina Veterinária registrados no Conselho Federal de Medicina Veterinária. Disponível em: <http://www.cfmv.org.br/portal/ensino_frm_exibir_opcao.php> acesso em 14 de mar. de 2020

Cipriani, J. (2019). Senado aprova PL que torna animais 'sujeitos'. Jornal Estado de Minas. Minas Gerais, p. $1-2.08$ ago. 08 Disponível https://www.em.com.br/app/noticia/politica/2019/08/08/interna politica,1075851/senado-aprovapl-que-torna-animais-sujeitos-mas-nao-barra-vaquejada.shtml. Acesso em: 08 ago. 2019.

Cohen, C., \& Regan, T. (2001). The animal rights debate. Rowman \& Littlefield.

CONCEA. Diretriz da prática de eutanásia do CONCEA 2015. Disponível em:< https://propesp.furg.br/images/arquivos_ceua/Resolucao-Normativa-n-37-Diretriz-da-Pratica-deEutanasia_site-concea.pdf >. Acesso em: 05 out. de 2020.

Cooney, K. A., Chappell, J. R., Callan, R. J., \& Connally, B. A. (2012). Veterinary euthanasia techniques: a practical guide. John Wiley \& Sons. DOI: https://doi.org/10.1002/9781118704585

Felix, Z. C., Costa, S. F. G., Alves, A. M. P. M., Andrade, C. G., Duarte, M. C. S., \& Brito, F. M. (2013). Eutanásia, distanásia e ortotanásia: revisão integrativa da literatura. Ciência \& Saúde Coletiva, 18, 2733-2746. DOI: https://doi.org/10.1590/s1413-81232013000900029

Garcia, A. L., Mesquita, J., \& Nóbrega, C. (2016). Cuidados paliativos em oncologia veterinária. Millenium-Journal of Education, Technologies, and Health, 14(37), 1-4.

Gustafson, D. L., \& Page, R. (2013). Saunders, Filadélfia, Pensilvânia, EUA. In S. J. Withrow, D. M. Vail, \& R. L. Page (Eds.), Small animal clinical oncology (pp. 157-179). Saunders Elsevier.

Hermes, H. R., \& Lamarca, I. C. A. (2013). Cuidados paliativos: uma abordagem a partir das categorias profissionais de saúde. Ciência \& Saúde Coletiva, 18, 2577-2588. DOI: https://doi.org/10.1590/S1413-81232013000900012 
IBGE - Instituto Brasileiro de Geografia e Estatística, 2013. Pesquisa Nacional por Amostra de Domicílios: lares com animais, 2013. Rio de Janeiro: IBGE

Lago, K. C. (2008). Fadiga por compaixão: quando ajudar dói. Universidade de Brasília.

Larue, S., \& Gordon, I. K. (2013). Radiation therapy. In S. J. Withrow \& E. G. Macewen (Eds.), Small animal clinical oncology (pp. 180-197). Sauders Elsevier. DOI: https://doi.org/10.1016/b978-14377-2362-5.00012-8

Lesnau, G. G., \& Santos, F. S. (2013). Formação dos acadêmicos de medicina veterinária no processo de morte e morrer. Bioscience Journal, 29(2), 429-433.

Marocchino, K. D. (2011). In the shadow of a rainbow: The history of animal hospice. Veterinary Clinics: Small Animal Practice, 41(3), 477-498. DOI: https://doi.org/10.1016/j.cvsm.2011.03.008

Polton, G. (2008). Getting started with veterinary chemotherapy. Irish Veterinary Journal, 61(7), 468-472.

Shanan, A., August, K., Cooney, K., Hendrix, L., Mader, B., \& Pierce, J. (2013). Animal Hospice and Palliative Care Guidelines. International Association of Animal Hospice and Palliative Care.

Shanan, A., \& Balasubramanian, V. (2011). Legal concerns with providing hospice and paliative care. Veterinary Clinics of the North America: Small Animal Practice, 41, 661-675. DOI: https://doi.org/doi10.1016/ j.cvsm.2011.03.007

Shearer, T. (2011a). Veterinary clinics of North America, small animal practice. In The role of the veterinarian in hospice and palliative care, 41(3) (pp. 11-13). DOI: https://doi.org/10.1016/j.cvsm.2011.03.018.

Shearer, T. (2011b). Veterinary clinics of North America: Small animal practice. In T. Shearer (Ed.), Pet hospice and palliative care protocols (pp. 507-518). DOI: https://doi.org/10.1016 / j.cvsm.2011.03.002

Villalobos, A. E. (2011). Quality-of-life assessment techniques for veterinarians. Veterinary Clinics: Small Animal Practice, 41(3), 519-529. DOI: https://doi.org/10.1016/j.cvsm.2011.03.013.

\section{Histórico do artigo:}

Recebido: 14 de outubro de 2020. Aprovado: 10 de novembro de 2020 Disponível online: 8 de fevereiro de 2021
Licenciamento: Este artigo é publicado na modalidade Acesso Aberto sob a licença Creative Commons Atribuição 4.0 (CC-BY 4.0), a qual permite uso irrestrito, distribuição, reprodução em qualquer meio, desde que o autor e a fonte sejam devidamente creditados. 